\title{
Population structure of Neisseria gonorrhoeae based on whole genome data and its relationship with antibiotic resistance
}

Matthew N. Ezewudo, Sandeep Joseph, Santiago Castillo-Ramirez, Deborah Dean, Carlos Del Rio, Xavier Didelot, Jo-Anne R Dillon, Richard F Selden, William M. Shafer, Rosemary S. Turingan, Magnus Unemo, Timothy D. Read

Neisseria gonorrhoeae is the causative agent of gonorrhea, a sexually transmitted infection (STI) of major importance. As a result of antibiotic resistance, there are now limited options for treating patients. We collected draft genome sequence data and associated metadata data on $76 \mathrm{~N}$. gonorrhoeae strains from around the globe and searched for known determinants of antibiotics resistance within the strains. The population structure and evolutionary forces within the pathogen population were analyzed. Our results indicated a cosmopolitan gonoccocal population mainly made up of five subgroups. The estimated ratio of recombination to mutation $(r / m=2.2)$ from our data set indicates an appreciable level of recombination occurring in the population. Strains with resistance phenotypes to more recent antibiotics (azithromycin and cefixime) were mostly found in two of the five population subgroups. 
3 Population structure of Neisseria gonorrhoeae based on whole genome

\title{
4 data and its relationship with antibiotic resistance
}

\author{
Matthew N. Ezewudoㅁ, Sandeep J. Joseph ${ }^{1}$, Santiago Castillo-Ramirez², Deborah Dean ${ }^{3}$, Carlos \\ del Rio ${ }^{1,4}$, Xavier Didelot ${ }^{5}$, Jo-Anne Dillon6, Richard F Selden ${ }^{7}$, William M. Shafer,9, Rosemary \\ S. Turingan ${ }^{7}$, Magnus Unemo ${ }^{10}$, *Timothy D. $\operatorname{Read}^{1}$ \\ $10{ }^{1}$ Department of Medicine, Division of Infectious Diseases, Emory University School of Medicine, \\ 11 Atlanta, Georgia, USA \\ 12 2Programa de Genómica Evolutiva, Centro de Ciencias Genómicas, Universidad Nacional Autónoma \\ 13 de México, Cuernavaca, Morelos CP 62210, México \\ $14{ }^{3}$ Children Hospital of Oakland Research institute, Oakland, California, USA \\ $15{ }^{4}$ Hubert Department of Global Health, Rollins School of Public Health of Emory University, Atlanta, \\ 16 Georgia, USA \\ 17 5epartment of Infectious Disease Epidemiology, Imperial College London, UK \\ $18{ }^{6}$ Department of Microbiology and Immunology, College of Medicine, Vaccine and Infectious Disease \\ 19 Organization International Vaccine Centre, University of, Saskatchewan, Saskatoon, Saskatchewan, \\ $21 \quad{ }^{7}$ NetBio, Waltham, MA USA \\ $22{ }^{8,9}$ Department of Microbiology and Immunology, Emory University School of Medicine, Atlanta, \\ 23 Georgia and Laboratories of Bacterial Pathogenesis, Veterans Affairs Medical Center, Decatur, \\ 24 Georgia, USA \\ $25{ }^{10} \mathrm{WHO}$ Collaborating Centre for Gonorrhea and other STIs, Örebro University Hospital, Örebro, \\ 26 Sweden \\ *Corresponding Author: Timothy D. Read \\ Department of Medicine, Division of Infectious Diseases, Emory University \\ School of Medicine, Atlanta, Georgia, USA tread@emory.edu +14047279706
}

20 Canada 


\section{Introduction}

35 Neisseria gonorrhoeae, a Gram-negative bacterium, causes gonorrhea, the most common bacterial

36 sexually transmitted infection (STIs) causing more than 106 million cases per year globally (World

37 Health Organization (WHO), 2012). The only effective option for treating the disease and stopping its

38 spread has been the use of antimicrobial therapy. Currently, there is no vaccine to prevent infection.

39 Antimicrobial treatment options have diminished over time due to the progressive emergence of

40 antimicrobial resistance (AMR) to drugs previously used to treat gonorrhea and the paucity in the

41 development of newer antibiotics that could effectively eradicate the pathogen (Ohnishi et al., 2011;

42 Unemo \& Shafer, 2014).

44 AMR evolution should be considered in the context of the genetic structure of the $N$. gonorrhoeae 45 population. Early work by O'Rourke et al. using electrophoretic analysis of enzymes of the pathogen 46 and serological methods suggested that gonococci have a non-clonal sexual or panmictic population 47 structure (O'Rourke \& Stevens, 1993). More recent studies have also suggested high rates of 48 recombination within the Neisseria genus (Didelot \& Maiden, 2010). High levels of recombination 49 could confound studies of the gonococcal populations, especially if the studies are based on few 50 genetic loci within strains as compared to the entire genomes. Recent multi-genome studies have

51 focused on either a restricted geographic region (Vidovic et al., 2014)(genomes also included in 52 present studies or on a small subset of the N. gonorrhoeae population (Grad et al., 2014). Hence, there 53 is a need for studies aimed at understanding the global $N$. gonorrhoeae population structure at the 54 whole genome scale.

56 Past AMR studies using limited numbers of gonococcal strains from specific geographic regions of the 
57 globe have mostly focused on a number of representative genes or genetic regions of the genome to

58 elucidate underlying mechanisms of antibiotic resistance (Hagman \& Shafer, 1995; Lindberg,

59 Fredlund, Nicholas, \& Unemo, 2007; Ohneck et al., 2011; Thakur, Levett, Horsman, \& Dillon, 2014;

60 Tomberg, Unemo, Ohnishi, Davies, \& Nicholas, 2013; Unemo \& Shafer, 2014; Unemo, Golparian, \&

61 Hellmark, 2014; Unemo, Golparian, \& Nicholas, 2012; Zhao et al., 2009). Extensive genome

62 sequencing studies have yet to be conducted on a diverse collection of strains from different

63 geographical locations and collected over longer time periods. Our approach in this study builds on

64 recent multi-genome studies (Grad et al., 2014; Vidovic et al., 2014), with the goal of using whole

65 genome analysis to elucidate two processes: 1) the population structure and dynamics of Neisseria

66 gonorrhoeae and 2) the correlation between this population differentiation and AMR evolution in

67 gonococci. Our genome analysis of strains from multiple sites across the world offers a geographic

68 diversity of $N$. gonorrhoeae isolates, providing more depth in genome-wide studies of this pathogen

69 and identifying possible sub-populations impacting AMR and evolution within the species.

\section{Materials and Methods}

\section{$71 \quad$ Neisseria gonorrhoeae isolates}

72 Sixty-one $N$. gonorrhoeae isolates of diverse origin were obtained. These included isolates from the

73 Gonococcal Isolation Surveillance Program (GISP) site covering Atlanta, Miami, New York city and

74 North Carolina in the United States $(\mathrm{n}=21)$, from Canada (primarily, Saskatchewan) and Chile $(\mathrm{n}=1)$

75 (Vidovic et al., 2014)) ( $\mathrm{n}=24)$, and from WHO global collaborations; Sweden ( $\mathrm{n}=7)$, Norway $(\mathrm{n}=3)$,

76 Japan ( $\mathrm{n}=2)$, Austria ( $\mathrm{n}=1)$, Pakistan ( $\mathrm{n}=1)$, Philippines $(\mathrm{n}=1)$, and Australia ( $\mathrm{n}=1)$. Phenotypic

77 determination of the minimum inhibitory concenterations (MICs) of all isolates was performed using

78 the agar dilution method or the Etest method (bioMerieux), according to the instructions from the 
79 manufacturer. The strains sequenced in this study were tested for resistance to primarily three

80 antibiotics, tetracycline, azithromycin and cefixime, with breakpoints for resistance set at 2, 2.0, and

$810.25 \mu \mathrm{g} / \mathrm{mL}$, respectively, based on the CDC MIC (minimum inhibitory concentration) breakpoints for

82 testing in the GISP protocol (http://www.cdc.gov/std/gisp/gisp-protocol07-15-2010.pdf). Antibiotic

83 resistance profiles of the Canadian strains have been previously reported (Vidovic et al., 2014). Details

84 of the different isolates with their NCBI accession numbers are presented in Table 1.

85

\section{Sequence generation and assembly}

87 The $N$. gonorrhoeae strains were shotgun (WGS) sequenced using the Illumina HiSeq ${ }^{\mathrm{TM}}$ instrument,

88 utilizing libraries prepared from $5 \mu \mathrm{g}$ of genomic DNA for each sample. The average sequencing

89 coverage was 225 . The sequencing reads were filtered using the prinseq-lite algorithm (Schmieder R.

90 et al, 2011) to ensure only sequence reads with average phred score $\geq 30$ were used. The reads for each

91 project were then assembled de novo, using the velvet assembler program (Zerbino \& Birney, 2008).

92 The optimal kmer length for each assembly prior to assembly was determined using the velvet

93 optimizer algorithm(Gladman \& Seemann, 2012). Data was deposited in the NCBI Sequence Read

94 Archive public database (Accession \# SRA099559) (Table 1). For this study, we included an additional

9514 draft genome sequences of $N$. gonorrhoeae strains, downloaded from the NCBI draft genomes

96 database (NCBI Bioproject numbers: PRJNA55649, PRJNA55651, PRJNA55653, PRJNA55905,

97 PRJNA46993, PRJNA55657, PRJNA55655, PRJNA55659, PRJNA55661, PRJNA55663,

98 PRJNA55665, PRJNA55667, PRJNA55669, PRJNA55671, and the reference genome sequence

99 Ref_FA_1090 (NC_002946.2) 
100 Genome-wide phylogeny and pangenome analysis

101 The assembled genomes were annotated individually using the NCBI PGAP annotation pipeline to give

102 predicted proteome for each of the strains. The orthologs were determined by OrthoMCL (Li,

103 Stoeckert, \& Roos, 2003), which uses bi-directional BLASTP scores of all the protein sequences to

104 perform Markov clustering in order to improve sensitivity and specificity of the orthologs. For the

105 OrthoMCL analysis, we used a BLASTP E-value cut-off of 1e-05, and inflation Markov clustering

106 parameter of 1.5. Core genes were defined as the orthologous genes that are shared among all the $N$.

107 gonorrhoeae strains used in this analysis

108

109 The nucleotide sequences of all the core genes were concatenated together and core-gene nucleotide

110 alignment was conducted using progressive MAUVE (Darling, Mau, Blattner, \& Perna, 2004).

111 Similarly, whole genome amino-acid alignment was also generated by concatenating the deduced

112 amino-acid sequences of all the core genes generated using MUSCLE(Edgar, 2004), and to form a

113 super protein alignment. Homoplasious sites were removed from the whole-genome nucleotide

114 alignment using the Noisy software (Dress et al., 2008). The protein alignments were filtered by

115 GBLOCKS (Talavera \& Castresana, 2007)using default settings to remove regions that contained gaps

116 or were highly diverged. A maximum likelihood (ML) tree from the same data set was created using

117 the GTR and JTT substitution models for the nucleotide and protein alignment respectively and the

118 GAMMA evolutionary model (Stamatakis, 2014). The majority rule-consensus tree was generated

119 from 200 bootstrap replicates of the model. Linear regression of the root-to-tip distances against the

120 year of isolation was performed using the Path-O-Gen tool

121 (http://tree.bio.ed.ac.uk/software/pathogen/).

122 


\section{Multi-locus sequence typing (MLST) locus analysis}

124 MLST is a genotyping tool for Neisseria based on sequencing of 7 core housekeeping genes(Jolley \&

125 Maiden, 2010). There are currently close to 11,000 individual Neisseria sequence profiles in the

126 publicly available MLST database (http://pubmlst.org). We utilized a custom python script

127 mlstBLAST.py (http://sourceforge.net/projects/srst/files/mlstBLAST/) to perform a BLAST search of

128 these genes across all the strains in our data set and identified the sequence type (ST) for each strain.

129 Novel alleles of the locus and STs were submitted to the MLST database. A phylogeny of the

130 concatenated DNA sequences of all the $N$. gonorrhoeae STs in the MLST public database was created

131 using the neighbor joining distance matrix approach of the PHYLIP(Felstein, 1989). Mean nucleotide

132 distance for the sequence alignments and MLST genes was computed using MEGA software (Tamura, 133 et al 2013).

\section{Estimating population parameters and homologous recombination}

136 ClonalFrame(Didelot \& Falush, 2007) utilizes a statistical framework to reconstruct the clonal

137 genealogy as well as identify the regions along the genomes that has been affected both by

138 recombination and mutation. The model uses a Bayesian approach to predict the phylogenetic

139 relationship in the sample set, given the whole genome sequence alignment data. The input genome

140 alignment data was the core genes $(\mathrm{n}=1189)$ alignment generated from MAUVE. Four independent

141 ClonalFrame runs were performed for 40,000 iterations, with the first 20,000 discarded as burn-in. This

142 allowed the model parameters to converge and each of the 4 runs were checked for the consistency of

143 the estimated parameters as well as the consistency of the topology of the inferred clonal genealogies.

\section{Population structure analysis}

145 The program BAPS (Bayesian Analysis of Population Structure) version 5.3 (Corander \& Marttinen, 
146 2006; Tang, Hanage, Fraser, \& Corander, 2009)was used to infer the underlying population structure of

147 the $76 \mathrm{~N}$. gonorrhoeae strains in the sample set. SNPs from the core MAUVE alignment, with gaps

148 removed were converted to a BAPS input file, which is a representation of all the polymorphic loci in

149 the multi-sequence alignment. BAPS applied a Bayesian model to predict the likelihood of a

150 population structure given the input data and non-parametric assumption approach to trace ancestry of

151 the different individuals in the sample set. For the mixture analysis we used the 'Clustering of

152 individuals' approach. We ran a preliminary analysis to evaluate the approximate number of

153 genetically differentiated groups using a vector from 2 to $40 \mathrm{~K}$ values, where $\mathrm{K}$ is the maximum

154 number of groups. Given that 5 groups was the $\mathrm{K}$ value with the best log likelihood, we ran a second

155 analysis using from 3 to $7 \mathrm{~K}$ values and again the best $\mathrm{K}$ value was 5 groups. We used the 'Admixture

156 based on mixture clustering' module for the admixture analysis. For the analysis; the minimum

157 population and the admixture coefficient for the individuals was then set to 5 . For the reference

158 individuals from each population and the admixture coefficient for reference individuals we used the

159 values as described by Castillo-Ramírez et al (Castillo-Ramírez et al., 2012). In addition, population

160 structure analysis of the sample set using the fineSTRUCTURE tool(Lawson, Hellenthal, Myers, \&

161 Falush, 2012) was performed. fineSTRUCTURE analysis was a two step process-1) ChromoPainter

162 algorithm was used to generate the co-ancestry matrix from the genome-wide haplotype data using the

163 linkage model. 2) The fineSTRUCTURE algorithm performed a model-based clustering using a

164 Bayesian MCMC approach to predict the likelihood of a population structure given the input data and

165 non-parametric assumption approach to trace ancestry of the different individuals in the sample set.

166 The fineSTRUCTURE approach was used to corroborate the findings from the BAPS population 167 structure analysis. 
170 We traced the flow of recombination between strains into five different subgroups in the phylogeny

171 determined from the subgroups of the population defined by the BAPS analysis. We created a BLAST

172 database of the whole genome sequence of all 76 strains in the sample set and included 14 whole

173 genome sequences of all other Neisseria species (NC_008767.1, NC_014752.1, NC_017512.1,

174 NC_017516.1, NC_003112.1, NC_010120.1 NC_017501.1, NC_017514.1, NC_017517.1,

175 NC_003116.1, NC_013016.1, NC_017505.1, NC_017515.1, NC_017518.1) that are present in the

176 NCBI database. Next, we performed a BLASTN search for each of the genomic region within the

177 strains identified by ClonalFrame to be under recombination, selecting the best hit within the sequences

178 in the database we created, with an identity of $>98 \%$, to be the source of the recombined region. We

179 also removed BLAST matches found in strains from similar subgroups as the source of the

180 recombined region. We used the migest package (http://cran.r-project.org/web/packages/migest/)

181 implemented in the $\mathrm{R}$ statistical language to create a circular representation of the matrix of

182 relationship between the subpopulations identified by BAPS based on the purported recombination

183 between strains in the different subgroups. We also supplied migest with the matrix from BAPS

184 admixture analysis and recreated the circular flow of recombination across only the subpopulations as 185 defined by BAPS.

\section{Comparison of nucleotide substitution rates}

189 Amino acid sequences were aligned using MUSCLE sequence aligner(Edgar, 2004). The amino acid 190 sequence alignment was converted to nucleotide alignment based on the corresponding gene sequence 191 using PAL2NAL(Suyama, Torrents, \& Bork, 2006) and we implemented the YN00 method of the 192 PAML package(Yang, 2007) to calculate the pairwise dN/dS ratios for the strains(Rocha et al., 2006).

193 The contribution of each strain to the overall variation in the $\mathrm{dN} / \mathrm{dS}$ rates across the sample set was 
194 estimated using ANOVA (Analysis of Variance) approaches.

\section{Analysis of positive selection}

197 For the analysis of positive selection within core genes of the strains in the sample set, we first

198 identified and removed core genes that have signals of homologous recombination using three methods

199 of Pairwise Homoplasy Index (PHI), Neighbor Similarity Score (NSS) and the maximum $\chi^{2}$ method.

200 The three methods are implemented in the PhiPack package(Sawyer, 1989). A window size of 50

201 nucleotides was used to run the methods in the package, and genes shown to have significant

202 probability of homologous recombination by a majority of the methods were not used for the positive

203 selection analysis. Next, we identified core genes under positive selection using codeml of PAML tool

204 version 4.7(Yang, 2007). We applied the branch-sites test for positive selection Model A test 2 of the

205 tool, to identify genes under positive selection population groups. For each of the clades, we performed

206 the Likelihood Ratio Test (LRT) for two hypotheses - the null hypothesis is the existence of neutral

207 selection as implemented in the null model versus the alternative hypothesis implemented in the test

208 model for positive selection. The LRT was performed to a degree of freedom of 1, and we corrected for

209 multiple testing using the False discovery rate approach (FDR)(Benjamini \& Hochberg, 1995). We

210 further identified the Gene Ontology (GO) terms and functional characterizations of the genes under

211 positive selection (see Table 2) and performed an enrichment test for functionality of these genes using

212 the blast2go test pipeline(Götz et al., 2011).

213

\section{Confirming known predictors of antibiotic resistance phenotype}

215 We downloaded from NCBI reference DNA sequences of resistance determinants that have been

216 shown in the literature to underlie the resistance phenotype we have observed in our sample set (see 
217 Table 3), and performed a BLASTN search for each of these DNA sequence regions across all the

218 strains in the database of whole genome sequences. For convenience, the contigs for each assembly

219 were ordered into one pseudocontig after tiling to the reference genome FA1090, using the ABACUS

220 tool (http://abacas.sourceforge.net/).

221 We selected the top hit (with identity match of $98 \%$ or more) for each sequence (strain) in the database 222 and parsed the alignment between the query and the subject sequence in the database for the presence 223 or absence of the underlying resistance genetic mutations as suggested in the literature.

\section{Results and Discussion}

\section{Genome-wide homologous recombination in diverse $N$. gonorrhoeae}

226 We sequenced 61 recent clinical isolates primarily from the US and Canada but also single

227 representatives from other countries, including Japan, Pakistan, Australia, Austria, Philippines, Norway

228 and Sweden using the WGS approach to a high average coverage (average 225-fold read redundancy).

229 De novo assemblies based on these data produced a set of contigs that represented draft, unordered

230 representations of the genomes with high sequence quality. A preliminary phylogeographical analysis

231 of the Canadian isolates ( $\mathrm{n}=23$ ) was recently published (Vidovic et al., 2014). For the analysis, we

232 included the $14 \mathrm{~N}$. gonorrhoeae draft NCBI genome sequences (12 from the US and 2 from Europe)

233 and the genome sequence of the FA1090 N. gonorrhoeae reference strain. The 76 were assigned into

23423 previously described MLST STs and four new STs $(10931,10932,10933,10934)$. The genetic

235 diversity (measured as pairwise nucleotide distances of MLST loci) of the strains in this study was

236 about half that of the $N$. gonorrhoeae strains as a whole ( 0.001 substitutions per site in our study

237 compared to 0.002 in the large MLST set), and the STs from our sample set were represented across

238 the different clades of a phylogeny of housekeeping genes of N. gonorrhoeae strains found in the 
239 MLST database (see Fig S5). Alignment of the shotgun assembly to reference genome FA1090

240 (NC_002946.2), yielded 10,962 SNPs in the core region (conserved in all strains). The average per

241 nucleotide diversity in the core genome regions was 0.003 .

243 Homologous recombination is known to play a role in shaping bacteria populations(Didelot \& Maiden, 244 2010). The ClonalFrame tool (Didelot \& Falush, 2007) detected 952 independent recombination

245 events, covering more than $50 \%$ of the reference genome. The average size of the recombination 246 regions identified was 360 base pairs. The estimate for the ratio of effects of recombination and 247 mutation $(r / m)$ for our strain set was 2.2, a relatively high value for bacterial species (Didelot \& 248 Maiden, 2010) (Fig S3, and quite similar to the $r / m$ estimate of 1.9 based on the whole genome 249 alignment on a less genetically diverse group of $N$. gonorrhoeae strains reported by Grad et al(Grad et 250 al., 2014).

252 We constructed a maximum likelihood phylogeny of the core genome of the 76 strains using the 253 RAxML program (excluding regions identified as potentially recombinant) (Fig 1). This tree had 254 similar topology to the clonal frame that determined by the eponymous software (Fig S3). The tree 255 showed multiple clades but the there was no strong signal of genetic isolation by distance at the 256 continental scale. The rate of the molecular clock was estimated to be $8.93 \times 10^{-6}$ mutations per year 257 based on the slope of the regression of the root-to-tip divergence with isolation dates (see Fig S2). This 258 value was similar to those obtained in other bacterial studies, ranging from $8.6 \times 10^{-9}$ to $2.5 \times 10^{-5}$ 259 (Zhou et al., 2013). However, because the temporal signal was weak in the root-to-tip analysis (Figure 260 S2), we did not use these data for Bayesian phylogeny analysis using the BEAST phylogeny tool 261 (Drummond \& Rambaut, 2007). 


\section{Neisseria gonorrhoeae population structure and biogeography}

265 Given that recombination was frequent in these genomes, we sought to evaluate the genetic

266 substructure of the population. We used two complementary methods. BAPS (Tang et al., 2009)

267 predicts the likelihood of a population structure given the input data and uses a non-parametric 268 assumption approach to trace ancestry. fineSTRUCTURE (Lawson et al., 2012), on the other hand, 269 uses similar methods of predicting population substructure, but to a finer detail and does not assume a 270 prior optimum number of subpopulations (K). The BAPS tool identified 5 subgroups within the $N$. 271 gonorrhoeae population from the strains within the sample set (Fig 3). As expected, members with the 272 same subgroup ancestry generally were found near each other when mapped on the ML phylogeny 273 constructed using the nonrecombining portion of the genome. On the other hand, fineSTRUCTURE identified 30 genetic subgroups within our sample set (Fig S4). However, individual members of each of the fineSTRUCTURE subgroups belong to the same BAPS subgroup. Each of the five BAPS subgroups contained strains from multiple continents based on geography or location of isolation (Fig 1). It was particularly interesting that each BAPS cluster had at least one US strain and one Canadian strain. The BAPS analysis revealed a complex relationship between Group 3 and 5, with the latter group 5 separated into two group 3 clades (Fig 1). Group 3 strains in clades closely related to group 5 showed significant genetic import from group 5. It is possible that the extent of admixture occurring in

281 group 3 and 5 may have caused misidentification. Also, strains of sequence type (ST) 1901, which is

282 the most abundant ST in our sample set all belong to subgroup 1, hinting at a correlation between 283 BAPS subgrouping and MLST.

We assessed patterns of genetic drift effects in the population by estimating the pairwise substitution rates between all the core gene orthologs for the strains and determining the mean $\mathrm{dN} / \mathrm{dS}$ ratio for each 
strain. The mean pairwise $\mathrm{dN} / \mathrm{dS}$ ratios for each strain are shown in Fig 4. There was significant

288

289

290

291

292

293

294

295

296

297

298

299

300

301

302

303

304

305

306

307

308

309

310

variation in the mean $\mathrm{dN} / \mathrm{dS}$ ratios among the strains (ANOVA p-value $=2.0 \mathrm{e}-16)$. The overall mean

of the $\mathrm{dN} / \mathrm{dS}$ estimate was 0.3184 , similar to the 0.402 value estimated for the bacterial pathogen

Chlamydia trachomatis (Joseph et al., 2012). The mean dN/dS ratio for the five subgroups respectively

was $(0.32412976,0.33325164,0.31273103,0.30952504$ and 0.30990092$)$. There is no significant

difference between group mean $\mathrm{dN} / \mathrm{dS}$ ratios ( $\mathrm{p}$-value $=0.921, \mathrm{t}$ test of means).

The mean $\mathrm{dN} / \mathrm{dS}$ ratio for strains from the Canadian region was 0.3279 , which was above the overall

mean ratio, while that for strains collected in the US was 0.31708 , which is below the overall pairwise

$\mathrm{dN} / \mathrm{dS}$ mean ratio for the sample set. This was also a statistically significant difference (p-value 0.0018

for $t$ test of means), suggesting a possible geographical effect within this subset of strains.

\section{Genetic admixture within $N$. gonorrhoeae and with other Neisseria species}

In order to understand the flow of genetic information between the strains from five different subgroups defined by the BAPS analysis (Fig 3) as well as strains from other Neisseria species, we used two independent approaches. The first was to search each of the 952 recombination regions identified by ClonalFrame for a best BLASTN match from another subgroup or Neisseria species (We created a blast database of the 76 genomes from this study and representative strains from the Neisseria genus) (Fig S3). In parallel, we also counted the occurrence of co-ancestry of genetic markers revealed by the BAPS analysis. Both the BAPS and BLAST analyses suggested that group 3 was the most common nexus of homologous recombination between other clades, consistent with its basal phylogenetic status. In the BAPS-based network groups 1 and 2 , and to a greater extent, group 5 , were primarily DNA donors to group 3 (Fig 2B). But this pattern was less visible in the BLAST network (Fig 2A). It is notable that more than $90 \%$ of the recombination with strains from other Neisseria species occured in groups 2 and 3. Group 5 stood out as a significant source of genetic exchange into 
311 strains in group 3.

312 The genetic relatedness of the strains in the sample set or the purported sharing of genetic materials

313 across the different subgroups shown by the BAPS figure paralleled the pattern revealed by the BLAST

314 clonal frame analysis ( $\mathrm{p}$-value $=0.048$, Mantel test for comparing the distance matrices of the five

315 populations between both methods). The exchange of genetic materials from other Neisseria species

316 was not accounted for in the BAPS admixture analysis. Based on the BLAST analysis, the proportion

317 of DNA transferred within N. gonorrhoeae compared to arriving from Neisseria strains the species was

318729 out of 849 intra-specific genetic events. This finding is line with the "fuzzy species" concept of

319 Fraser et al(Fraser, Hanage, \& Spratt, 2007): while N. gonorrhoeae is not sexually isolated, DNA flow

320 seemed predominantly through intra-specific exchanges.

\section{Genes under positive selection}

324 Of the 1189 core genes, we identified 352 genes as likely to contain past recombination histories using 325 the PHIPACK tests (Sawyer, 1989). Thirty-one genes within the subset of 837 non-recombining core 326 genes were found to be under positive selection using the tests implemented by the PAML software 327 (Materials and Methods). BAPS subgroup 5 had the highest number of core genes under selection (14)

328 followed by subgroup 3 (7). While we found no significant enrichment of genes under positive

329 selection in any of the functional classes in the Gene Ontology (GO) database, the functions of the best

330 match proteins from genes under positive selection can be broadly classified to genes involved in DNA

331 or RNA synthesis of gene expression, membrane or transport proteins, and, to a lesser extent, genes

332 involved in metabolic pathways in the bacterial cell (Supplement Data S2 spreadsheet). Of the 352

333 genes found to have signals of recombination, we found no significant enrichment of the genes in any

334 of the functional classes in the GO database. The functions of these genes could broadly be classified 
335 into 2 groups: genes encoding membrane and transport proteins; and those involved in metabolic 336 pathways in the cell (Supplement Data S3).

338 In regard to antibiotic resistance and selection, the most interesting gene found to be under positive 339 selection was porB (Smith, Maynard Smith, \& Spratt, 1995), which has been shown to be involved in

340 mechanisms of resistance to penicillins, macrolides, cephalosporins and tetracyclines (Unemo \&

341 Shafer, 2014). porB exhibited signals of selection in subgroups 1, 3 and 5 - the groups that harbored

342 most of the antibiotic resistant strains in our sample set (Fig 5). comA, which encodes a membrane

343 protein necessary for competence of $N$. gonorrhoeae, was also found to be under selection in a handful

344 of strains that make up subgroup 5. This finding is of interest in regards to the potential for DNA

345 uptake in these strains, since they appear to be primarily DNA donors, rather than recipients in genetic

346 exchanges (Fig 2). Other genes putatively under selection included a stress response gene, a gene

347 encoding a chaperone protein of the HscA family and a number of proteins: ribosyl transferase, RNA

348 polymerase, and an ars $R$ family transcriptional regulator, which were all linked to gene expression.

349 Genes under positive selection in subgroup 3 were also mainly involved in gene expression or DNA

350 metabolism, including DNA helicase and tRNA pseudo uridine synthase. Most of the genes with

351 known functions, identified to be under positive selection in subgroup 1 were either membrane-

352 associated or transport proteins.

\section{Analysis of known genetic predictors for AMR phenotypes}

354 A substantial amount of research effort over the past 10 years has been devoted to understanding the

355 genetic basis of drug resistance in N. gonorrhoeae (Garvin et al., 2008; MD et al., 2014; Unemo \&

356 Shafer, 2011; Veal, Nicholas, \& Shafer, 2002; World Health Organization (WHO), 2012). Since there

357 is a an increasing interest in the direct attribution of resistance phenotypes based on genome 
358 sequencing, we attempted to ascertain how knowledge of existing variants could be applied to the $N$.

359 gonorrhoeae genomes in this study. We searched for variants known to underlie resistance to 3

360 antibiotics classes within our study (Table 3). In terms of subgroup distribution, tetracycline resistance

361 was found in each of the 5 population subgroups, azithromycin resistance was present in only 2 of the

362 strains tested (SK36809 and MUNG8) and restricted to subgroup 2 (Fig 5), and cefixime resistance

363 was found in subgroup 1 and subgroup 2. We identified genes responsible for resistance to the drugs

364 tested in this works using literature searches and the CARD antimicrobial resistance database

365 (McArthur et al., 2013).

367 The tetM resistant determinant, which confers high-level resistance to tetracycline, is borne on

368 plasmids and is transferred either through conjugation or transformation (Knapp, Johnson, Zenilman,

369 Roberts, \& Morse, 1988; Morse, Johnson, Biddle, \& Roberts, 1986; Turner, Gough, \& Leeming,

370 1999). It was found in only 5 of the 10 strains with high-level resistance to tetracyline (MIC equal or

371 greater than $16 \mu \mathrm{g} / \mathrm{ml}$ ). Strain SK1902, one of the 5 strains with the tet $M$ determinant, had a

372 significantly higher MIC ( $>256 \mu \mathrm{g} / \mathrm{ml})$ than the rest (see attached Supplement S3). Other strains with

373 reduced susceptibility or chrosomally-mediated resistance to tetracycline, i.e without the tetM

374 determinant do have other corresponding chromosomal mutations on one or more of the resistance loci:

$375 m t r R$ (including its promoter), penB, rpsJ. Only one strain (ATL0508) within the sample set exhibits

376 resistance to tetracycline in the laboratory, without the presence of any of the known resistance

377 determinants of the tetracycline resistance phenotype.

379 Different "mosaic" penA alleles are thought to have developed from recombination with portions of

380 DNA transferred horizontally from commensal Neisseria and/or N. meningitidis and underly decreased

381 susceptibility or resistance to cephalosporins by preventing their binding action on the encoded mosaic 
382 PBP2 (Ameyama et al., 2002). The mosaic penA XXXIV (Ohnishi, 2011; Grad 2014; Unemo \&

383 Shafer, 2014) had the best positive predictive value of all the known resistance determinants we

384 searched for within our dataset, being present in $6 / 7$ of the strains resistant to cefixime. This result

385 echoed the observations made by Grad et al (Grad et al., 2014)in their epidemiologic study of $N$.

386 gonorrhoeae strains. The other loci (i.e., mutations in the $m t r R, m t r C D E$ operon promoter region and

387 penB gene) also proven to enhance the MICs of cephalosporins (Unemo \& Shafer, 2011; Warner,

388 Shafer, \& Jerse, 2008) did not have a similar predictive property within strains in our data set. These

389 variants were seen in 2 out of 7 and 3 out of 7 cefixime resistant strains, respectively. MUNG17 is the

390 only strain in the sample set that has an elevated MIC $(0.38 \mu \mathrm{g} / \mathrm{mL})$ to cefixime that we could not find

391 any of the known resistance determinants within its genome sequence (See attached supplement Data 392 S1).

393

394 Resistance to azithromycin can be mediated by mutations in the previously mentioned penB and $m t r$ 395 operon genes as well as mutations found in the 4 different alleles of the 23S rRNA gene that inhibits 396 protein synthesis (Chisholm et al., 2009; Palmer, Young, \& Winter, 2008; Starnino, Stefanelli,

397 Neisseria gonorrhoeae Italian Study Group, 2009). The 23S rRNA mutation allele was found in one 398 (SK36809) of the two strains with the azithromycin resistance phenotype. The other azithromycin 399 resistant strain, MUNG8, did not have the 23S rRNA resistance determinant or any of the other known 400 mutations in the $m$ trR or penB loci (See attached Supplement Data S1).

401

\section{Conclusions}

403 Our study suggested that $N$. gonorrhoeae globally is made up of at least five genetic subpopulations.

404 That individual strains from the subpopulations are from diverse geographical locations confirms the 
405 cosmopolitan nature of the pathogen. This suggested a population structure with multiple waves of 406 rapid international expansion. Subgroup 3 strains may be the nexus for gene exchange within the 407 species. Groups 1 and 2 might be the most recently branched and contain a higher proportion of 408 resistant isolates to more currently used antibiotics. Given the importance of the antibiotic resistant 409 phenotype, these may be emerging lineages that are expanding within $N$. gonorrhoeae. It will require a 410 more extensive study with a broader number of strains to ascertain this suggested evolutionary trend.

411 Our analysis confirms earlier studies that showed an appreciable effect of recombination within the 412 population. This could be playing a role in the evolution of AMR in the bacterium, as strains with 413 resistance phenotypes to currently used antibiotics are mostly within similar population sub-groupings.

415 Although most of the known predictors that underlie the observed resistance phenotypes were 416 accounted for in the strains we studied, they could not explain some of the phenotypes of several 417 strains. These findings suggested that a broader genome search of a large number of whole genomes 418 from strains of this pathogen could yield candidate novel variants that may explain some of the 419 "missing" antibiotic resistance phenotypes we have observed.

420 In general, large genome sequencing studies examining a high number of temporally and

421 geographically diverse $N$. gonorrhoeae isolates are essential to elucidate the evolution and diversity of 422 the $N$. gonorrhoeae species as well as associations between genomic content, antibiotic resistance and 423 clinical outcome of treatment.

\section{Acknowledgements}

427 Genome sequencing was performed at the Emory Genomics Center. We wish to thank Tauqeer Alam 
at Emory University, for advice on phylogenetic methods, and Sinisa Vidovic and Sidarath Dev from the Vaccine and Infectious Disease Orgarnization- International Vaccine center at the University of Saskatchewan, Canada for their help in antimicrobial susceptibility determination and preliminary genomic assessments. We also thank the Broad Institute for pre-publication release of genomic data (Bioproject PRJNA55649- PRJNA55649) used in this study.

\author{
(Bioproject PRJNA55649- PRJNA55649) used in this study.
}

(1)

(1)

6

7


Noisy: identification of problematic columns in multiple sequence alignments. Algorithms for molecular biology : $A M B, 3,7$. doi:10.1186/1748-7188-3-7

Drummond, A. J., \& Rambaut, A. (2007). BEAST: Bayesian evolutionary analysis by sampling trees. $B M C$ evolutionary biology, 7, 214. doi:10.1186/1471-2148-7-214

Edgar, R. C. (2004). MUSCLE: a multiple sequence alignment method with reduced time and space complexity. BMC Bioinformatics, 5, 113-113. doi:10.1186/1471-2105-5-113

Felstein, J. (1989). PHYLIP-Phylogeny Inference Package. Cladistics, 5(2), 163-166. doi:10.1111/j.10960031.1989.tb00562.x

Fraser, C., Hanage, W. P., \& Spratt, B. G. (2007). Recombination and the nature of bacterial speciation. Science, 315(5811), 476-480. doi:10.1126/science.1127573

Garvin, L. E., Bash, M. C., Keys, C., Warner, D. M., Ram, S., Shafer, W. M., \& Jerse, A. E. (2008). Phenotypic and Genotypic Analyses of Neisseria gonorrhoeae Isolates That Express Frequently Recovered PorB PIA Variable Region Types Suggest that Certain P1a Porin Sequences Confer a Selective Advantage for Urogenital Tract Infection. Infection and Immunity, 76(8), 3700-3709. doi:10.1128/IAI.00265-08

Gladman, S., \& Seemann, T. (2012, December 22). Velvet Optimiser. vicbioinformatics.com. Retrieved May 30, 2014, from http://www.vicbioinformatics.com/software.velvetoptimiser.shtml

Götz, S., Arnold, R., Sebastián-León, P., Martín-Rodríguez, S., Tischler, P., Jehl, M.-A., Dopazo, J., Rattei, T., \& Conesa, A. (2011). B2G-FAR, a species-centered GO annotation repository. Journal of Gerontology, 27(7), 919-924. doi:10.1093/bioinformatics/btr059

Grad, Y. H., Kirkcaldy, R. D., Trees, D., Dordel, J., Harris, S. R., Goldstein, E., Weinstock, H., Parkhill, J., Hanage, W.P., Bentley, S., Lipsitch, M. (2014). Genomic epidemiology of Neisseria gonorrhoeae with reduced susceptibility to cefixime in the USA: a retrospective observational study. The Lancet Infectious Diseases, 14(3), 220-226. doi:10.1016/S1473-3099(13)70693-5

Hagman, K. E., \& Shafer, W. M. (1995). Transcriptional control of the mtr efflux system of Neisseria gonorrhoeae. Journal of bacteriology, 177(14), 4162-4165.

Jolley, K. A., \& Maiden, M. C. J. (2010). BIGSdb: Scalable analysis of bacterial genome variation at the population level. BMC Bioinformatics, 11, 595. doi:10.1186/1471-2105-11-595

Joseph, S. J., Didelot, X., Rothschild, J., de Vries, H. J. C., Morré, S. A., Read, T. D., \& Dean, D. (2012). Population genomics of Chlamydia trachomatis: insights on drift, selection, recombination, and population structure. Molecular Biology and Evolution, 29(12), 3933-3946. doi:10.1093/molbev/mss 198

Knapp, J. S., Johnson, S. R., Zenilman, J. M., Roberts, M. C., \& Morse, S. A. (1988). High-level tetracycline resistance resulting from TetM in strains of Neisseria spp., Kingella denitrificans, and Eikenella corrodens. Antimicrobial Agents and Chemotherapy, 32(5), 765-767.

Lawson, D. J., Hellenthal, G., Myers, S., \& Falush, D. (2012). Inference of population structure using dense haplotype data. PLoS genetics, 8(1), e1002453. doi:10.1371/journal.pgen.1002453

Li, L., Stoeckert, C. J., \& Roos, D. S. (2003). OrthoMCL: identification of ortholog groups for eukaryotic genomes. Genome Research, 13(9), 2178-2189. doi:10.1101/gr.1224503

Lindberg, R., Fredlund, H., Nicholas, R., \& Unemo, M. (2007). Neisseria gonorrhoeae isolates with reduced susceptibility to cefixime and ceftriaxone: association with genetic polymorphisms in penA, mtrR, porB1b, and ponA. Antimicrobial Agents and Chemotherapy, 51(6), 2117-2122. doi:10.1128/AAC.01604-06

McArthur, A. G., Waglechner, N., Nizam, F., Yan, A., Azad, M. A., Baylay, A. J., Bhullar, K., Canova, M. J., De Pascale, G., Ejim, L., Kalan, L., King, A. M., Koteva, K., Morar, M., Mulvey, M. R., O'Brien, J. S., Pawlowski, A. C., Piddock, L. J. V., Spanogiannopoulos, P., Sutherland, A. D., Tang, I., Taylor, P. L., Thaker, M., Wang, W. L., Yan, M., Yu, T., \& Wright, G. D. (2013). The comprehensive antibiotic resistance database. Antimicrobial Agents and Chemotherapy, 57(7), 3348-3357. doi:10.1128/AAC.00419-13

Morse, S. A., Johnson, S. R., Biddle, J. W., \& Roberts, M. C. (1986). High-level tetracycline resistance in Neisseria gonorrhoeae is result of acquisition of streptococcal tetM determinant. Antimicrobial Agents and Chemotherapy, 30(5), 664-670.

O'Rourke, M., \& Stevens, E. (1993). Genetic structure of Neisseria gonorrhoeae populations: a non-clonal pathogen. Journal of general microbiology, 139(11), 2603-2611.

Ohneck, E. A., Zalucki, Y. M., Johnson, P. J. T., Dhulipala, V., Golparian, D., Unemo, M., Jerse A.E., \& Shafer 
W. M. (2011). A novel mechanism of high-level, broad-spectrum antibiotic resistance caused by a single base pair change in Neisseria gonorrhoeae. MBio, 2(5). doi:10.1128/mBio.00187-11

Ohnishi, M., Golparian, D., Shimuta, K., Saika, T., Hoshina, S., Iwasaku, K., Nakayama, S, Kitawaki, J., \& Unemo, M. (2011). Is Neisseria gonorrhoeae Initiating a Future Era of Untreatable Gonorrhea?: Detailed Characterization of the First Strain with High-Level Resistance to Ceftriaxone. Antimicrobial Agents and Chemotherapy, 55(7), 3538-3545. doi:10.1128/AAC.00325-11

Palmer, H. M., Young, H., \& Winter, A. (2008). Emergence and spread of azithromycin-resistant Neisseria gonorrhoeae in Scotland. Journal of antimicrobial chemotherapy, 62(3), 490 -494.

Rocha, E. P. C., Smith, J. M., Hurst, L. D., Holden, M. T. G., Cooper, J. E., Smith, N. H., \& Feil, E. J. (2006). Comparisons of $\mathrm{dN} / \mathrm{dS}$ are time dependent for closely related bacterial genomes. Journal of theoretical biology, 239(2), 226-235. doi:10.1016/j.jtbi.2005.08.037

Sawyer, S. (1989). Statistical tests for detecting gene conversion. Molecular Biology and Evolution, 6(5), 526538.

Smith, N. H., Maynard Smith, J., \& Spratt, B. G. (1995). Sequence evolution of the porB gene of Neisseria gonorrhoeae and Neisseria meningitidis: evidence of positive Darwinian selection. Molecular Biology and Evolution, 12(3), 363-370.

Stamatakis, A. (2014). RAxML version 8: a tool for phylogenetic analysis and post-analysis of large phylogenies. Bioinformatics. doi:10.1093/bioinformatics/btu033

Starnino, S., Stefanelli, P., Neisseria gonorrhoeae Italian Study Group. (2009). Azithromycin-resistant Neisseria gonorrhoeae strains recently isolated in Italy. The Journal of antimicrobial chemotherapy, 63(6), 12001204. doi:10.1093/jac/dkp118

Suyama, M., Torrents, D., \& Bork, P. (2006). PAL2NAL: robust conversion of protein sequence alignments into the corresponding codon alignments. Nucleic Acids Research, 34(Web Server issue), 609-612. doi:10.1093/nar/gk1315

Talavera, G., \& Castresana, J. (2007). Improvement of phylogenies after removing divergent and ambiguously aligned blocks from protein sequence alignments. Systematic Biology, 56(4), 564-577. doi:10.1080/10635150701472164

Tang, J., Hanage, W. P., Fraser, C., \& Corander, J. (2009). Identifying currents in the gene pool for bacterial populations using an integrative approach. PLoS computational biology.

Thakur, S. D., Levett, P. N., Horsman, G. B., \& Dillon, J.-A. R. (2014). Molecular epidemiology of Neisseria gonorrhoeae isolates from Saskatchewan, Canada: utility of NG-MAST in predicting antimicrobial susceptibility regionally. Sexually transmitted infections, 90(4), 297-302. doi:10.1136/sextrans-2013051229

Tomberg, J., Unemo, M., Ohnishi, M., Davies, C., \& Nicholas, R. A. (2013). Identification of amino acids conferring high-level resistance to expanded-spectrum cephalosporins in the penA gene from Neisseria gonorrhoeae strain H041. Antimicrobial Agents and Chemotherapy, 57(7), 3029-3036. doi:10.1128/AAC.00093-13

Turner, A., Gough, K. R., \& Leeming, J. P. (1999). Molecular epidemiology of tetM genes in Neisseria gonorrhoeae. Sexually transmitted infections, 75(1), 60-66.

Unemo, M., \& Shafer, W. M. (2011). Antibiotic resistance in Neisseria gonorrhoeae: origin, evolution, and lessons learned for the future. Annals of the New York Academy of Sciences, 1230(1), E19-E28. doi:10.1111/j.1749-6632.2011.06215.x

Unemo, M., \& Shafer, W. M. (2014). Antimicrobial resistance in Neisseria gonorrhoeae in the 21st century: past, evolution, and future. Clinical Microbiology Reviews, 27(3), 587-613. doi:10.1128/CMR.00010-14

Unemo, M., Golparian, D., \& Hellmark, B. (2014). First three Neisseria gonorrhoeae isolates with high-level resistance to azithromycin in Sweden: a threat to currently available dual-antimicrobial regimens for treatment of gonorrhea? Antimicrobial Agents and Chemotherapy, 58(1), 624-625. doi:10.1128/AAC.02093-13

Unemo, M., Golparian, D., \& Nicholas, R. (2012). High-level cefixime-and ceftriaxone-resistant Neisseria gonorrhoeae in France: novel penA mosaic allele in a successful international clone causes treatment failure. Antimicrobial agents and Chemotherapy 56(3) 1273 - 1280 
Veal, W. L., Nicholas, R. A., \& Shafer, W. M. (2002). Overexpression of the MtrC-MtrD-MtrE efflux pump due to an mtrR mutation is required for chromosomally mediated penicillin resistance in Neisseria gonorrhoeae. Journal of bacteriology, 184(20), 5619-5624.

Vidovic, S., Caron, C., Taheri, A., Thakur, S. D., Read, T. D., Kusalik, A., \& Dillon, J.-A. R. (2014). Using Crude Whole-Genome Assemblies of Neisseria gonorrhoeae as a Platform for Strain Analysis: Clonal Spread of Gonorrhea Infection in Saskatchewan, Canada. Journal of Clinical Microbiology, 52(10), 37723776. doi:10.1128/JCM.01502-14

Warner, D. M., Shafer, W. M., \& Jerse, A. E. (2008). Clinically relevant mutations that cause derepression of the Neisseria gonorrhoeaeMtrC-MtrD-MtrE Efflux pump system confer different levels of antimicrobial resistance and in vivofitness. Molecular Microbiology, 70(2), 462-478. doi:10.1111/j.13652958.2008.06424.x

World Health Organization (WHO). (2012). Global action plan to control the spread and impact of antimicrobial resistance in Neisseria gonorrhoea.

Yang, Z. Z. (2007). PAML 4: phylogenetic analysis by maximum likelihood. Molecular Biology and Evolution, 24(8), 1586-1591. doi:10.1093/molbev/msm088

Zerbino, D. R., \& Birney, E. (2008). Velvet: algorithms for de novo short read assembly using de Bruijn graphs. Genome Research, 18(5), 821-829. doi:10.1101/gr.074492.107

Zhao, S., Duncan, M., Tomberg, J., Davies, C., Unemo, M., \& Nicholas, R. A. (2009). Genetics of chromosomally mediated intermediate resistance to ceftriaxone and cefixime in Neisseria gonorrhoeae. Antimicrobial Agents and Chemotherapy, 53(9), 3744-3751. doi:10.1128/AAC.00304-09

Zhou, Z., McCann, A., Litrup, E., Murphy, R., Cormican, M., Fanning, S., Brown, D., Guttman, D., S., Brisse, S., \& Achtman, M.. (2013). Neutral Genomic Microevolution of a Recently Emerged Pathogen, Salmonella enterica Serovar Agona. PLoS genetics, 9(4), e1003471. doi:10.1371/journal.pgen.1003471 


\section{1}

Phylogeny of $N$. gonorrhoeae strains in the sample set

(A) (Top). Maximum Likelihood phylogeny of sequenced strains of N. gonorrhoeae Branches with boostrap value $>80 \%$ for branches are indicated. Taxa are highlighted based on 5 different subgroups defined by BAPS. Annotations next to the leaves are colored based on location of isolation; Canada is colored red, US blue, Europe green, Asia purple and the lone strains from Australia (MUNG6) and Chile (CH811) is colored brown. (B) Unrooted phylogeny based on the same tree. 


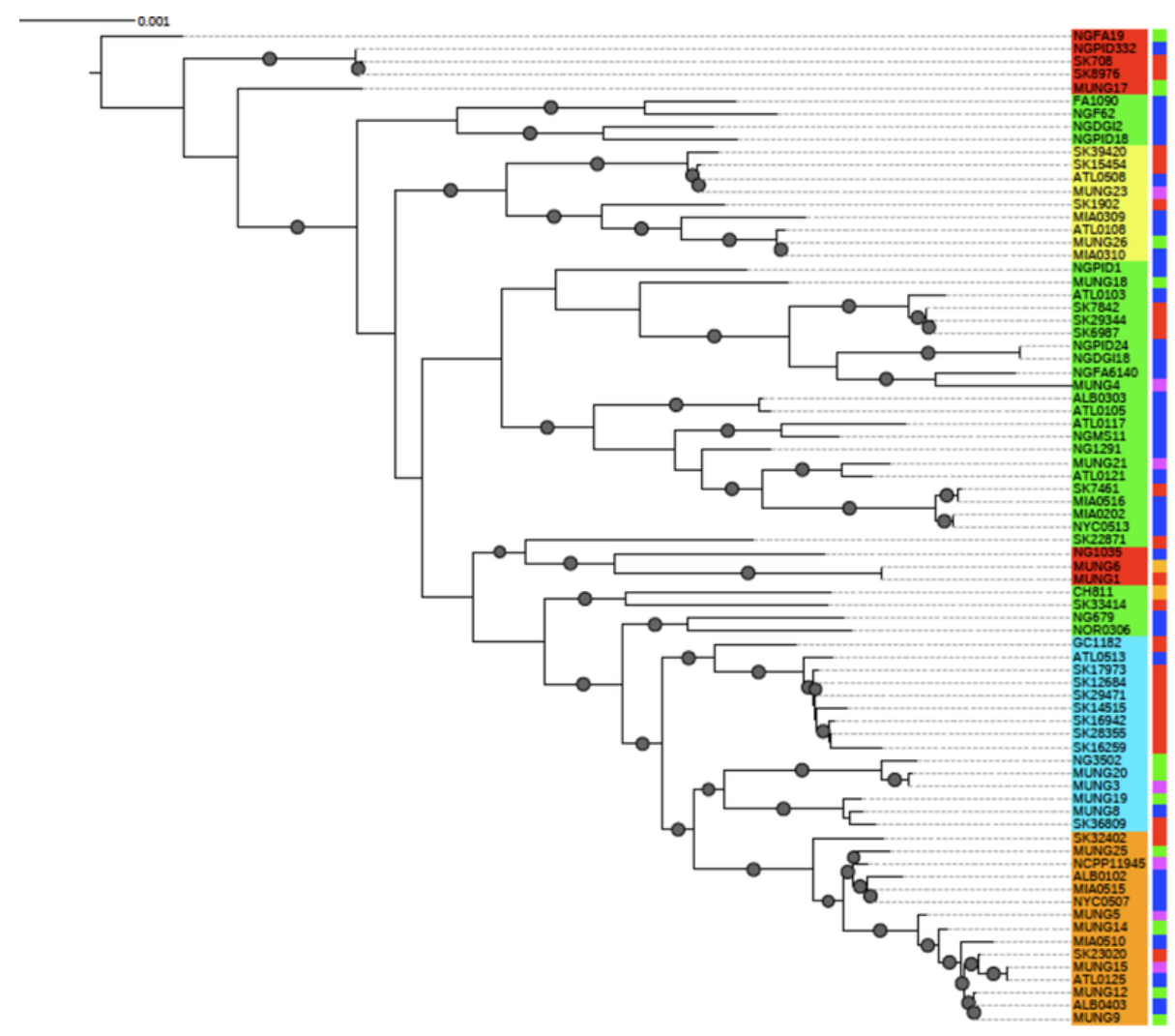

A

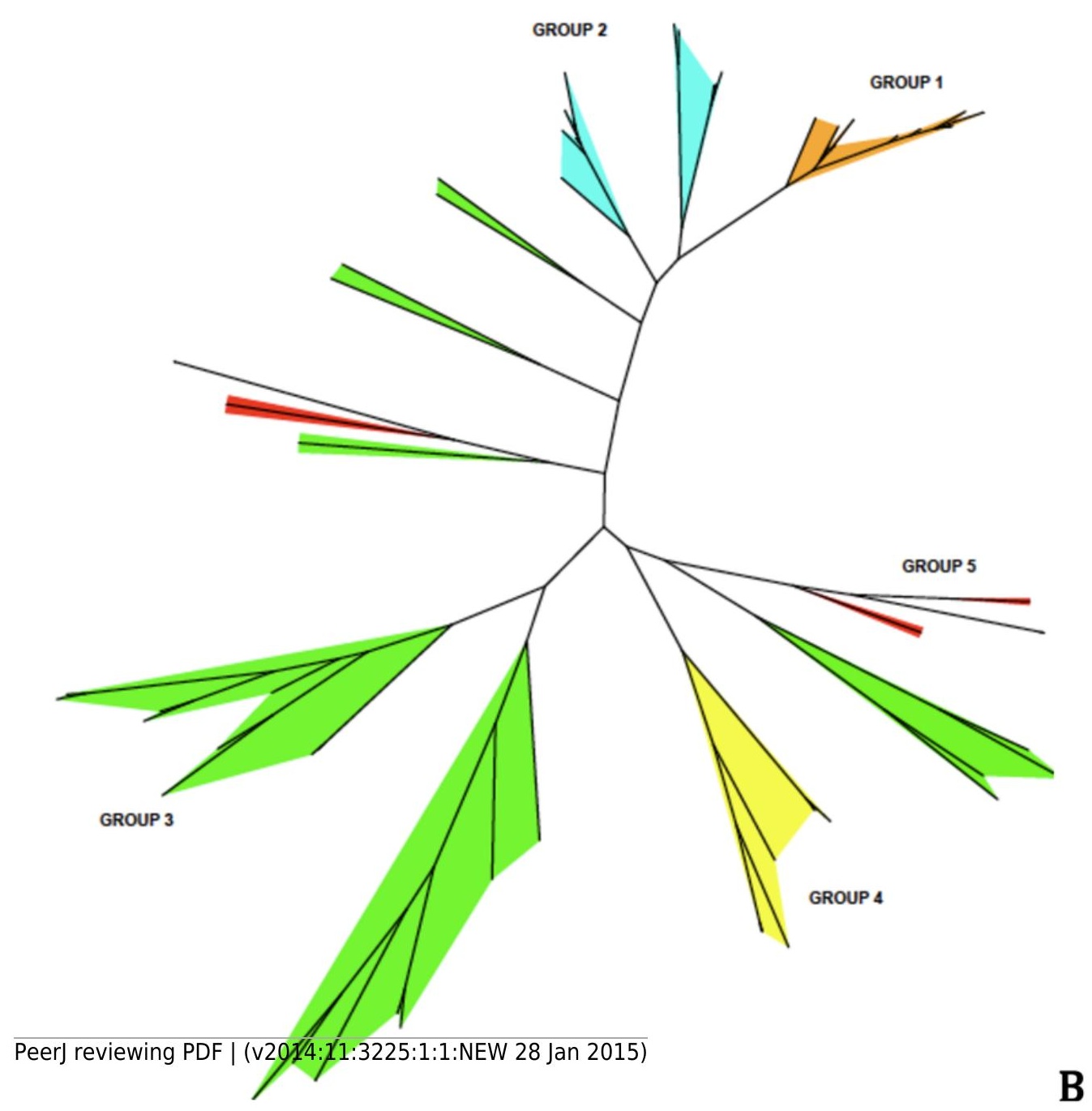


2

Pathways for exchange of geneticmaterials between populations

. (A) Recombination pattern traced from BLAST results of similarity of recombined regions between the subgroups defined by BAPS of $N$. gonorrhoeae. The clade showed as external represents strains from other Neisseria species. (B) Exchange of genetic materials among subgroups within the sample set as defined by BAPS admixture analysis. Colored base subsectors of the circle for each subgroup in the diagram represents outflow of genetic material while blank or white colored sub-sectors represent inflow of genetic materials to the subgroups. The figure was created using the migest package of the R statististical tool. 


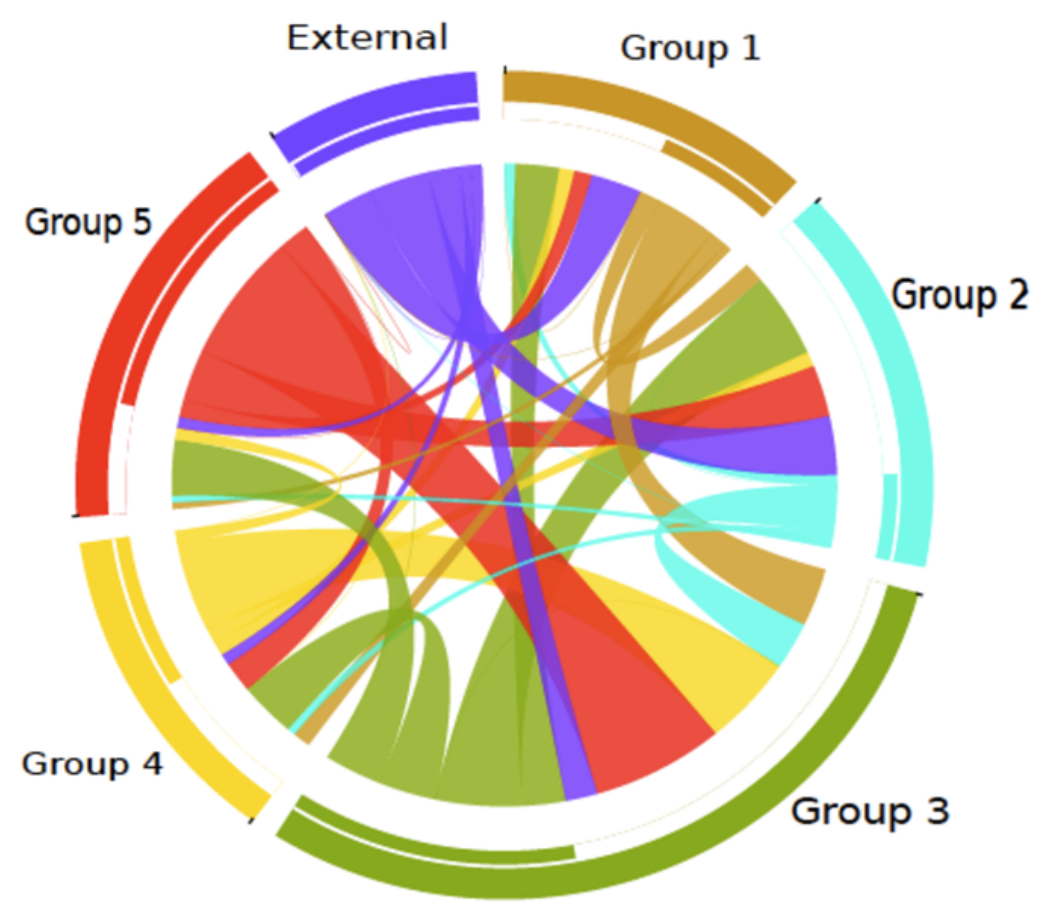

A

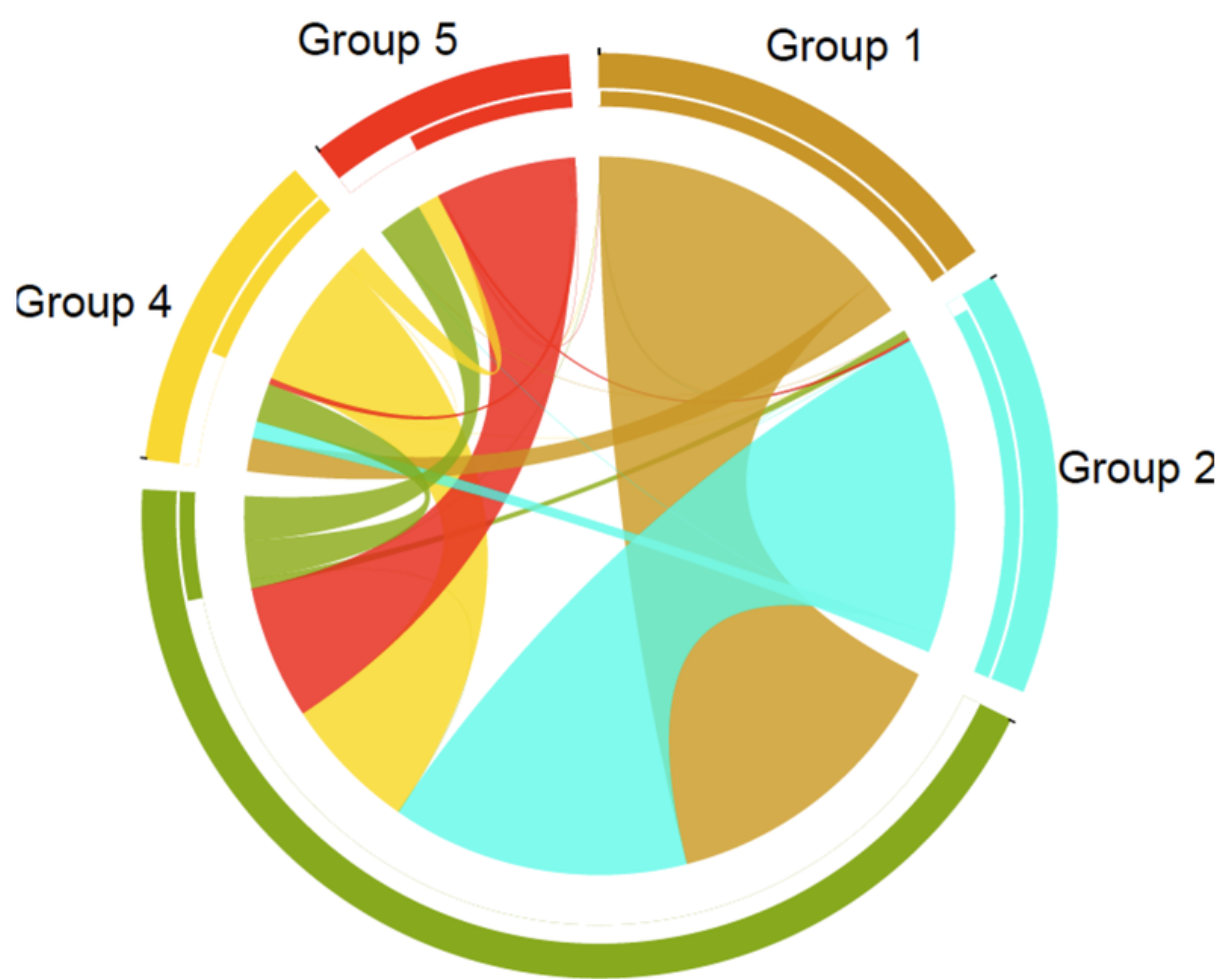




\section{3}

Population subgroups from strains or N. gonorrhoeae in the sample set defined by BAPS

The names for each strain in the different subgroup are indicated at the bottom of the plot on the $x$-axis, while the fineSTRUCTURE group labels for each strain is indicated on top of the plot. This figure shows that the structure of the Neisseria gonorrhoeae population divides into five subgroups. Each color represents one of the genetically differentiated groups and each vertical colored bar corresponds to one isolate. When the vertical bars show two colors, each color corresponds to one of the groups and this is evidence for admixture; the proportion of every color in the bar reflects the proportion of the genome coming from the group represented by that particular color.

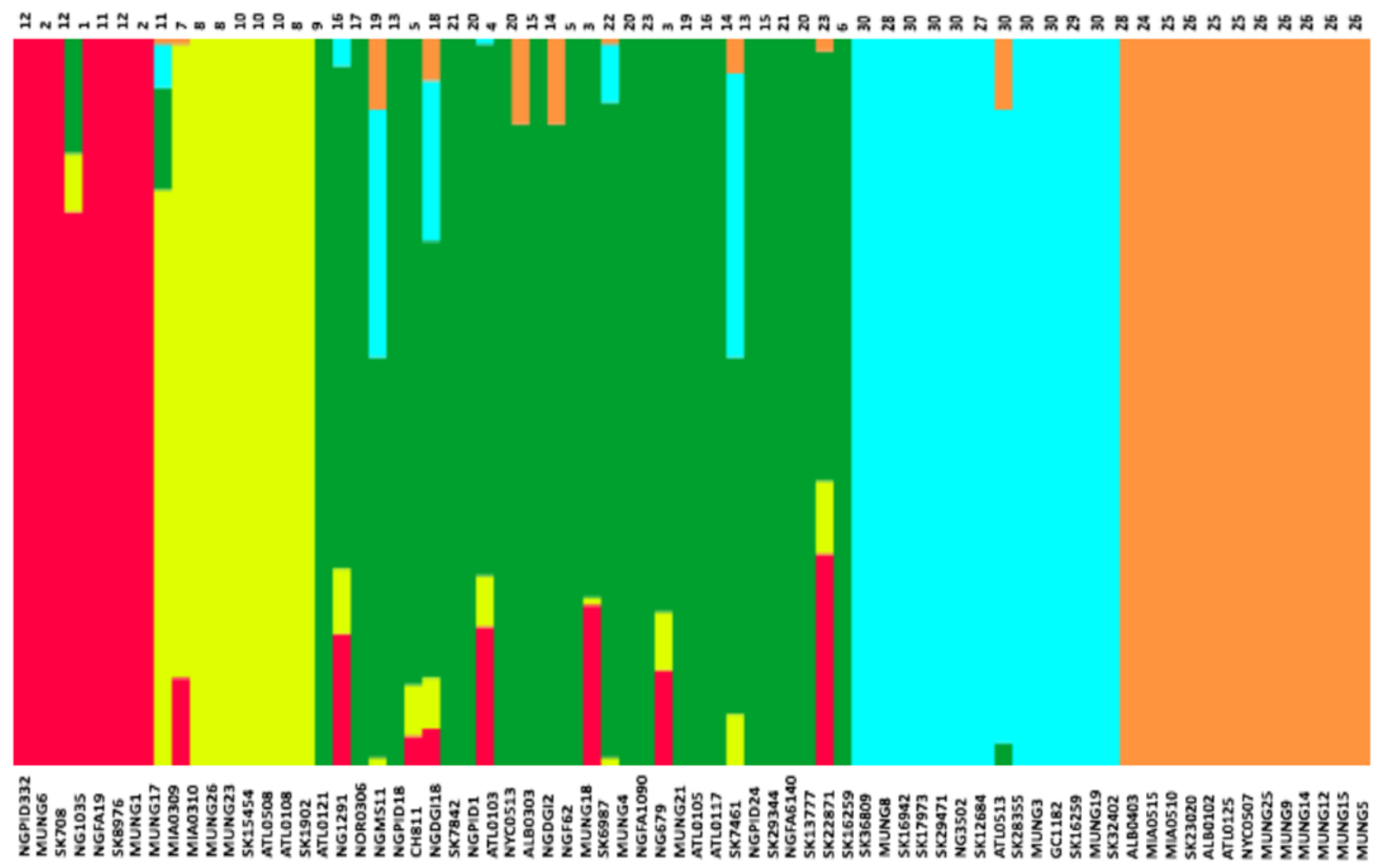


4

Boxplot of mean dN/dS ratio pair-wise comparison of core genes of each of the strains of N. gonorrhoeae in the sample set

The box plot is colored by subgroups within the Neisseria population, defined by the BAPS tool.

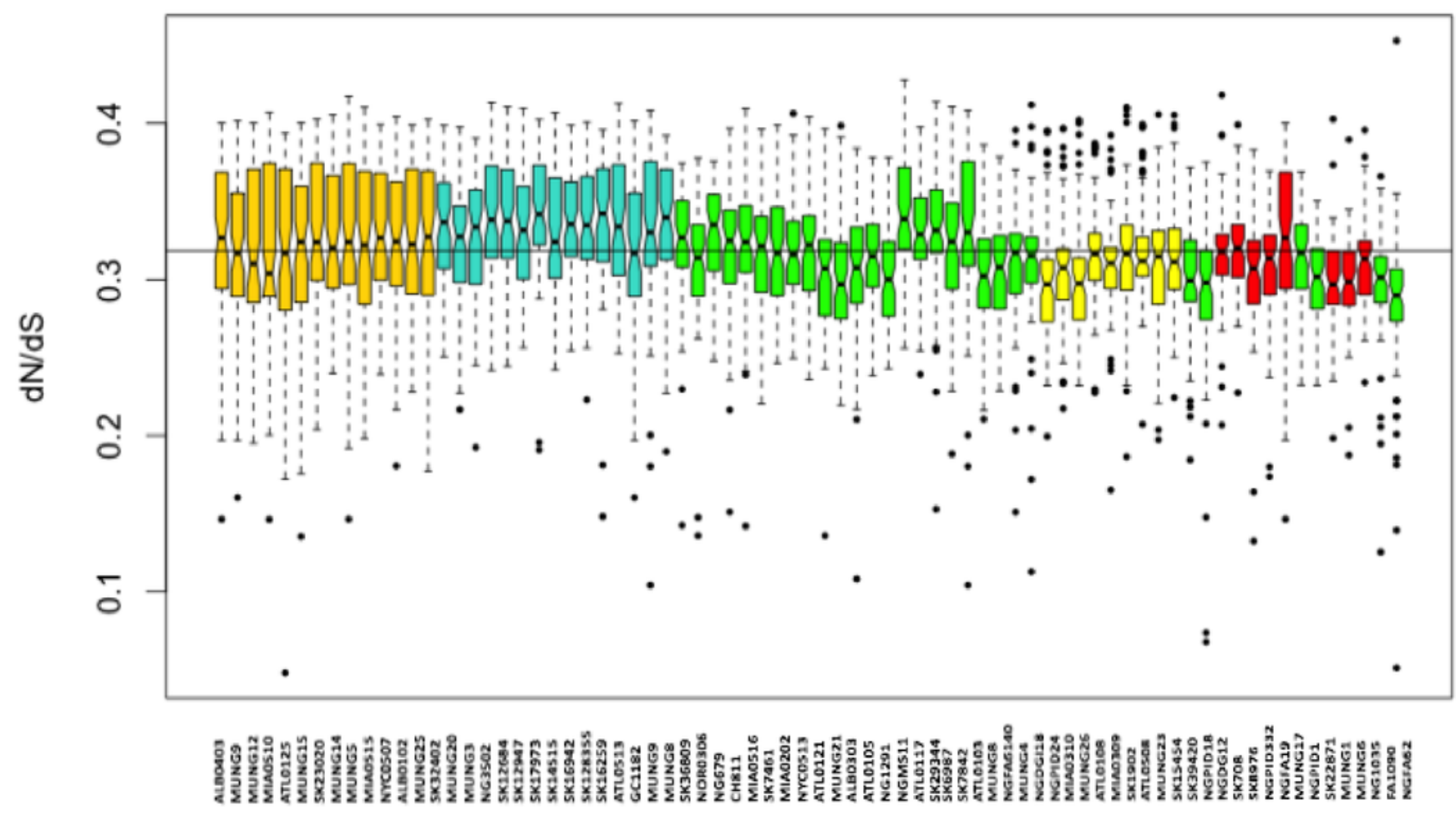




\section{5}

Representation of antibiotic resistance profile of $N$. gonorrhoeae strains across different subgroups of the population

The topology is identical to the $M L$ tree in Figure 1.

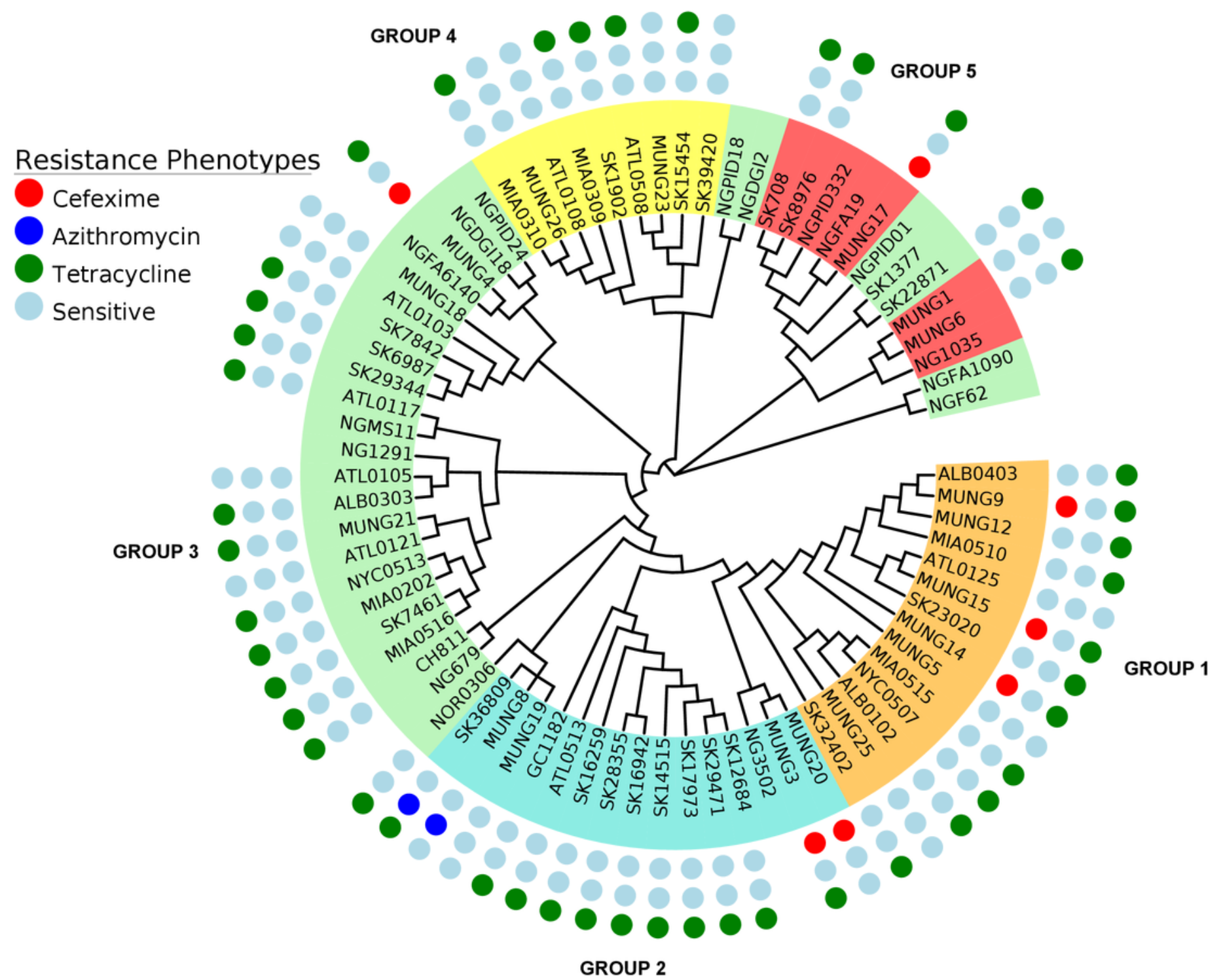




\section{Table $\mathbf{1}$ (on next page)}

Location and date of collection of the $N$. gonorrhoeae strains including Sequence Types and MICs of the different strains to the antibiotics azithromycin, cefixime and tetracycline.

The MIC breakpoint value for azithromycin resistance is $2 \mu \mathrm{g} / \mathrm{mL}$, for cefixime $0.25 \mu \mathrm{g} / \mathrm{mL}$, and for tetracycline $2 \mu \mathrm{g} / \mathrm{mL}$, based on the CDC breakpoints for antibiotic testing. 


\begin{tabular}{|c|c|c|c|c|c|c|}
\hline $\begin{array}{l}\text { Strain } \\
\text { Name }\end{array}$ & Location & Date & MLST & $\begin{array}{l}\text { Azithromycin } \\
\text { (MIC) }\end{array}$ & $\begin{array}{l}\text { Cefixime } \\
\text { (MIC) }\end{array}$ & $\begin{array}{l}\text { Tetracycline } \\
\text { (MIC) }\end{array}$ \\
\hline CH811 & Chile & 1982 & 1583 & 0.25 & 0.008 & 2 \\
\hline GC1-182 & Canada & 1982 & 1583 & 0.5 & 0.008 & 4 \\
\hline SK708 & Canada & 2006 & 1594 & 1 & 0.016 & 0.5 \\
\hline SK1902 & Canada & 2006 & 10935 & 0.25 & 0.002 & 256 \\
\hline SK6987 & Canada & 2006 & 10010 & 1 & 0.016 & 4 \\
\hline SK7461 & Canada & 2008 & 1901 & 0.5 & 0.032 & 8 \\
\hline SK7842 & Canada & 2006 & 10010 & 1 & 0.016 & 8 \\
\hline SK8976 & Canada & 2006 & 1594 & 0.06 & 0.004 & 2 \\
\hline SK12684 & Canada & 2006 & 31129 & 0.5 & 0.016 & 8 \\
\hline SK33414 & Canada & 2007 & 1928 & 0.25 & 0.008 & 4 \\
\hline SK14515 & Canada & 2005 & 1893 & 0.25 & 0.016 & 2 \\
\hline SK15454 & Canada & 2007 & 1585 & 0.06 & 0.004 & 2 \\
\hline SK16259 & Canada & 2007 & 1893 & 0.125 & 0.008 & 4 \\
\hline SK16942 & Canada & 2005 & 1893 & 0.125 & 0.016 & 2 \\
\hline SK17973 & Canada & 2006 & 1893 & 1 & 0.016 & 8 \\
\hline
\end{tabular}




\begin{tabular}{|c|c|c|c|c|c|c|}
\hline SK22871 & Canada & 2007 & 8122 & 0.125 & 0.004 & 4 \\
\hline SK23020 & Canada & 2006 & 1901 & 0.25 & 0.125 & 16 \\
\hline SK28355 & Canada & 2007 & 1893 & 0.25 & 0.016 & 4 \\
\hline SK29344 & Canada & 2007 & 10010 & 0.125 & 0.008 & 4 \\
\hline SK29471 & Canada & 2005 & 1893 & 0.25 & 0.016 & 2 \\
\hline $\mathrm{SK}_{32402}$ & Canada & 2007 & 8153 & 0.5 & 0.016 & 4 \\
\hline SK36809 & Canada & 2007 & 8126 & 2 & 0.008 & 8 \\
\hline SK3942O & Canada & 2008 & 1585 & 0.5 & 0.016 & 0.5 \\
\hline ALBo303 & USA & 2011 & 1588 & 0.03 & 0.015 & 16 \\
\hline ALB0403 & USA & 2011 & 1901 & 1 & 0.125 & 4 \\
\hline ATL0103 & USA & 2011 & 10931 & 0.5 & 0.015 & 0.25 \\
\hline ALBo102 & USA & 2011 & 1901 & 0.25 & 0.06 & 2 \\
\hline ATL0105 & USA & 2011 & 1588 & 0.06 & 0.015 & 0.25 \\
\hline ATL0108 & USA & 2011 & 1584 & 0.03 & 0.015 & 0.25 \\
\hline ATL0117 & USA & 2011 & 10932 & 0.125 & 0.015 & 16 \\
\hline ATL0121 & USA & 2011 & 1902 & 0.5 & 0.03 & 1 \\
\hline ATL0125 & USA & 2011 & 1901 & 0.25 & 0.015 & 1 \\
\hline ATL0508 & USA & 2011 & 1585 & 0.06 & 0.015 & 16 \\
\hline
\end{tabular}




\begin{tabular}{|c|c|c|c|c|c|c|}
\hline ATLO513 & USA & 2011 & 1893 & 0.25 & 0.03 & 2 \\
\hline MIA0202 & USA & 2011 & 1901 & 0.5 & 0.03 & 2 \\
\hline MIA0309 & USA & 2011 & 1931 & 0.125 & 0.015 & 16 \\
\hline MIA0310 & USA & 2011 & 1584 & 0.03 & 0.015 & 16 \\
\hline MIAo510 & USA & 2011 & 1901 & 1 & 0.03 & 2 \\
\hline MIAO515 & USA & 2011 & 1901 & 0.25 & 0.03 & 16 \\
\hline MIAO516 & USA & 2011 & 1901 & 0.5 & 0.06 & 8 \\
\hline NORo306 & USA & 2011 & 1583 & 0.25 & 0.015 & 2 \\
\hline NYCo507 & USA & 2011 & 1901 & 0.25 & 0.06 & 2 \\
\hline NYCo513 & USA & 2011 & 1901 & 0.25 & 0.06 & 4 \\
\hline MUNG1 & Canada & 1991 & 10934 & 0.125 & $<0.016$ & 0.25 \\
\hline MUNG3 & Japan & 2003 & 7363 & 0.25 & 0.5 & 2 \\
\hline MUNG4 & Japan & 1996 & 1590 & 0.5 & 0.25 & 4 \\
\hline MUNG5 & $\begin{array}{l}\text { Philippin } \\
\text { es }\end{array}$ & 1992 & 1901 & 0.25 & $<0.016$ & 1 \\
\hline MUNG6 & Australia & 2001 & 10008 & 0.125 & $<0.016$ & 16 \\
\hline MUNG8 & USA & 2001 & 8127 & 2 & $<0.016$ & 0.5 \\
\hline MUNG9 & Sweden & 2010 & 1901 & 0.5 & 1 & 2 \\
\hline
\end{tabular}




\begin{tabular}{|c|c|c|c|c|c|c|}
\hline MUNG12 & Norway & 2010 & 1901 & 0.5 & 0.25 & 4 \\
\hline MUNG14 & Norway & 2010 & 1901 & 0.5 & 0.25 & 4 \\
\hline MUNG15 & Austria & 2011 & 1901 & 0.25 & 1 & 2 \\
\hline MUNG17 & Sweden & 2010 & 1892 & 1 & 0.5 & 2 \\
\hline MUNG18 & Norway & 2010 & 10933 & 0.125 & $<0.016$ & 2 \\
\hline MUNG19 & Sweden & 2010 & 1580 & $>256$ & $<0.016$ & 2 \\
\hline MUNG2O & Sweden & 2013 & 7363 & 0.25 & 0.5 & 2 \\
\hline MUNG21 & Pakistan & 2008 & 1902 & 1 & 0.032 & 2 \\
\hline MUNG23 & Sweden & 1998 & 1585 & 0.064 & $<0.016$ & 0.125 \\
\hline MUNG25 & Sweden & 1998 & 1901 & 0.125 & $<0.016$ & 0.5 \\
\hline MUNG26 & Sweden & 1999 & 1584 & 0.064 & $<0.016$ & 0.5 \\
\hline
\end{tabular}


Table 2 (on next page)

Core genes of $N$. gonorrhoeae under positive selection in the different clades of the phylogeny of strains in the sample set 


\begin{tabular}{|c|c|c|}
\hline Gene & Clades Present & $\begin{array}{l}\text { Gene ID (reference } \\
\text { genomeFA 1090) }\end{array}$ \\
\hline PorB & $1,3,5$ & NGO1812 \\
\hline Acetate kinase 2 & 5 & NGO1521 \\
\hline $\begin{array}{l}\text { Primosomal replication } \\
\text { protein }\end{array}$ & 3 & NGOo582 \\
\hline DNA Helicase & 3,5 & NGO1196 \\
\hline Hypothetical protein & 5 & NGOo880 \\
\hline Hypothetical Protein & 5 & NGO1847 \\
\hline Hypothetical protein & 5 & NGO1948 \\
\hline ComA & 5 & NGO0276 \\
\hline Chaperone Protein HscA & 5 & NGOo829 \\
\hline tRNA-ribosyltransferase & 5 & NGOo294 \\
\hline RNA polymerase Subunit $\beta$ & 5 & NGO1850 \\
\hline $\begin{array}{l}\text { ArsR family transcriptional } \\
\text { regulator }\end{array}$ & 5 & NGO1562 \\
\hline Hypothetical protein & 5 & NGO0165 \\
\hline PriB & 5 & NGOo582 \\
\hline $\mathrm{ABC}$ transporter subunit & 3 & NGO2088 \\
\hline Hypothetical protein & 3 & NGO1984 \\
\hline $\begin{array}{l}\text { tRNA pseudouridine } \\
\text { synthase B }\end{array}$ & 3 & NGOo642 \\
\hline Prolyl endopeptidase & 1 & NGOoO26 \\
\hline Apo-lipoprotein N- & 1 & NGO0289 \\
\hline
\end{tabular}




\begin{tabular}{|l|l|l|}
\hline acyltransferase & & \\
\hline Sodium dependent & 1 & NGO2096 \\
\hline Phansporter & & \\
\hline Hypothetical Protein & 4 & NGO1012 \\
\hline
\end{tabular}

3

4 
Table 3 (on next page)

Known antibiotic resistance determinants in sample set

The description includes the PubMed reference ID and associated resistance phenotypes of these determinants in $\mathrm{N}$. gonorrhoeae. Cephalosporin antibiotics include cefixime and ceftriaxone, while macrolides include erythromycin and azithromycin. 


\begin{tabular}{|c|c|c|c|c|}
\hline Gene name & $\begin{array}{l}\text { FA1o9o } \\
\text { Reference } \\
\text { locus_tag/G } \\
\text { ene Bank ID }\end{array}$ & $\begin{array}{l}\text { Genetic } \\
\text { Mutations }\end{array}$ & $\begin{array}{l}\text { Resistance } \\
\text { Phenotype }\end{array}$ & References \\
\hline$m t r \mathrm{R}$ & NGO1366 & $\begin{array}{l}\text { G45D, A39T } \\
\text { (glycine and } \\
\text { aspartate } \\
\text { substitutions) }\end{array}$ & $\begin{array}{l}\text { Decreased } \\
\text { susceptibility to } \\
\text { macrolides and } \\
\text { beta-lactams }\end{array}$ & $\begin{array}{l}\text { PMID: } \\
18761689\end{array}$ \\
\hline $\begin{array}{l}m t r C D E \\
\text { promoter }\end{array}$ & NGO1366 & $\begin{array}{l}\text { Single } \\
\text { nucleotide } \\
\text { deletion on } \\
\text { reference } \\
\text { genome } \\
\text { position } \\
1327932\end{array}$ & $\begin{array}{l}\text { Decreased } \\
\text { susceptibility to } \\
\text { macrolides and } \\
\text { beta-lactams }\end{array}$ & $\begin{array}{l}\text { PMID: } \\
18761689\end{array}$ \\
\hline pen $\mathrm{B}$ & NGO1812 & $\begin{array}{l}\text { G101K, A102D } \\
\text { (glycine and } \\
\text { alanine } \\
\text { substitutions) }\end{array}$ & $\begin{array}{l}\text { Decreased } \\
\text { susceptibility to } \\
\text { third-generation } \\
\text { cephalosporins }\end{array}$ & $\begin{array}{l}\text { PMID: } \\
17420216\end{array}$ \\
\hline Mosaic penA & NGO1542 & $\begin{array}{l}\text { Mosaic } \\
\text { pattern amino } \\
\text { acid } \\
\text { substitutions } \\
\text { form position } \\
294 \text { to end of } \\
\text { gene }\end{array}$ & $\begin{array}{l}\text { Decreased } \\
\text { susceptibility to } \\
\text { third-generation } \\
\text { cephalosporins }\end{array}$ & $\begin{array}{l}\text { PMID: } \\
20028823\end{array}$ \\
\hline$r p s \mathrm{~J}$ & NGO1841 & V57M & $\begin{array}{l}\text { Decreased } \\
\text { susceptibility to } \\
\text { tetracycline }\end{array}$ & $\begin{array}{l}\text { PMID:16189 } \\
114\end{array}$ \\
\hline 23S rRNA & $\mathrm{AF} 450080$ & $\begin{array}{l}\text { C2611T } \\
\text { (Cystine to } \\
\text { Threonine } \\
\text { substitution) }\end{array}$ & $\begin{array}{l}\text { Decreased } \\
\text { susceptibility to } \\
\text { Azithromycin }\end{array}$ & $\begin{array}{l}\text { PMID: } \\
12183262\end{array}$ \\
\hline tet $\mathrm{M}$ & $\mathrm{N} / \mathrm{A}$ & $\begin{array}{l}\text { Horizontally } \\
\text { transferred } \\
\text { determinant } \\
\text { on plasmids }\end{array}$ & $\begin{array}{l}\text { Resistance to } \\
\text { tetracycline (MIC } \\
>=16 \mu \mathrm{g} / \mathrm{ml} \text { ) }\end{array}$ & $\begin{array}{l}\text { PMID: } \\
21349987\end{array}$ \\
\hline
\end{tabular}

